\title{
SIGNIFICANCE OF COGNITIVE ASSESSMENT IN RELATION TO OCCUPATIONAL THERAPY PRACTICE, AN EXPLORATORY STUDY FOR PAKISTAN OCCUPATIONAL THERAPY GUIDELINES
}

\section{ABSTRACT \\ BACKGROUND AND AIMS}

Cognition is defined as the ability to perceive process and comprehend the information from the surrounding. Impairment in cognitive skills can significantly affect individual's performance. The objective of the study is to identify the commonly used cognitive assessment tools by occupational therapist and determine its significance in occupational therapy practice.

\section{METHODOLOGY}

A total of 150 participants were enrolled in the crosssectional survey that was responded by occupational therapist working in pediatric domain specifically in outpatient rehabilitation setting. The self-structured questionnaire was validated by factor analysis through SPSS.

\section{RESULTS:}

The findings of this study revealed that $96 \%$ of occupational therapists performed cognitive assessment out of which only $9.6 \%$ occupational therapist administer standardized assessments due to incompetency and lack of resources the rest use informal mode of assessments. Mini mental state examination (MMSE) is found to be most common cognitive assessment tool while tools like Loewenstein Occupational Therapy Cognitive Assessment (LOTCA) is rarely used though it is very significant for Activities of daily living (ADL) cognition

\section{CONCLUSION}

It was concluded that the need of cognitive assessment in pediatric setting is an integral component in occupational therapy process for authentic evaluation and effective intervention plan. The curriculum must promote efficient training of standardize assessment and resources should be provided for better outcome and prognosis.

\section{KEYWORDS}

Cognition, Assessment, Occupational Therapists, Rehabilitation, Interview, standardized tool, MMSE, LOTCA
Aliza Imtiaz

Occupational Therapist

Karachi Down Syndrome program

Alizaimtiaz15@gmail.com
[Imtiaz A. Significance Of Cognitive Assessment In Relation To Occupational Therapy Practice, An Exploratory Study For Pakistan Occupational Therapy Guidelines Pak.j. rahbil.2021;10(1): 17-25] DOI: 10.36283/pjr.zu.10.1/004 


\section{INTRODUCTION}

Cognition is an ability of an individual to perceive and process information from the surrounding. It includes attention, memory, judgment, thinking, and observation that significantly affect emotion and behavior. It can also be defined as building-up information learned through daily experiences and learning which helps the individual to understand the world around. Individual's ability of comprehension and interaction with the environment is influenced by its culture, norms, values, and personality and past experiences. Cognitive processes are fundamental aspect of one's adaptive functioning in the environment. Cognition and memory make base for adaptive functioning and movements. It is also important for visual memory, perception and comprehension of language which is crucial for communication and builds foundation for motor planning and interaction with the surrounding ${ }^{1}$.

The American Occupational Therapy Association (AOTA) emphasizes that occupational therapists and occupational therapy assistants use the occupations (meaningful activities of life that occupies ones time) and activities to facilitate individuals' cognitive functioning for improvement of occupational performance, self-reliance, participation in community, and improve overall health and wellbeing ${ }^{2}$. Cognitive abilities are crucial for the effective functioning in daily occupations of life such as work and rest, education, home management, play and leisure. It also plays an essential role in human growth and development, and in the ability to acquire, recall, and use new information in response to changes in everyday life ${ }^{2}$. The evaluation of cognitive abilities of an individual determines its capability to live independently, perform meaningful life roles, function adaptively in the environment and adapt new routines and habits $^{3}$. Researches shows that cognitive abilities are influenced by biopsycho-social interaction, individual learning experiences and culture ${ }^{4}$.

Cognition can also be defined as process of acquiring and processing information from surrounding through the use of senses and use it in daily experiences. It encompasses high intellectual abilities such as attention, memory, planning and problem solving, reasoning and judgment, comprehension and language and decision making. Cognitive impairment is the term that defines impairments of intellectual abilities ${ }^{5}$. There are many causes that can be linked to cognitive impairment. It can be either syndromic (neurocognitive disorder) or etiologic (early symptoms of Alzheimer's disease). Studies also shows that after age of 70 years, $16 \%$ of elderly population develop symptoms of mild cognitive impairment and dementia is diagnosed in $14 \%$ of older people ${ }^{6}$.

Daily living and instrumental activities of life may get affected by the limitation of cognition affecting overall wellbeing. Such deficiencies may leads to adverse consequences in performing meaningful tasks. Occupational therapy has essential function in a multidisciplinary team approach to promote individual's daily performance and participation in various contexts to improve health and wellbeing, and quality of life by addressing cognitive impairment. $^{7}$

Occupational therapy practitioners assert that cognitive functioning can only be understood and facilitated fully within the context of occupational performance. This phenomenon of the connection between the client, his or her roles, daily occupations, and context make occupational therapy a profession that is exclusively qualified to address cognitive impairments and improve client's performance $^{8}$.

The cognitive assessment can be defined as the assessment or evaluation of 
cognitive abilities through questionnaire, interview or observation used for screening $^{9}$. It is inexpensive, non-invasive and quick method to identify individuals at risk of developing cognitive impairments or to identify the level of cognitive decline ${ }^{10}$. The cognitive assessment tools assist the therapist to recognize the client's functional status and level of impairment, also to make intervention goals and evaluate progress ${ }^{11}$.

Occupational therapist performs cognitive assessments through standardized and non-standardized assessments tools. The standardized assessments (e.g. MiniMental State Examination ${ }^{12,}$ Loewenstein Occupational Therapy Cognitive Assessment-Geriatrics ${ }^{13}$ ) are formal assessments that are developed by evaluating thousands of individual to set the norm. The administration and scoring is done on the defined pattern while, the non-standardized assessment is an informal assessment which does not have strict criteria of administration and evaluation.

The standardized and non-standardized tools are utilized for the assessment of specific cognitive and perceptual disabilities. On the other hand, cognitive capacities, memory and problem-solving strategies are also surveyed focusing on their roles based on occupational performance, observation and formal interviews. The standardized assessments utilize quantitative information with other experts on the health-care team. These assessments established for evidencebased practice to compare clients in intervention comprised of a task to be performed by the patients, questionnaire/exercise to be completed by patients and scored by the therapist. Nonstandardized assessments include observations and interviews that are most commonly used to gather information on

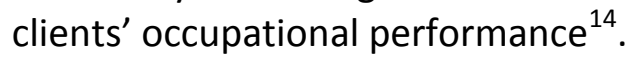

According to American Occupational Therapy Association referred (AOTA) cognitive instruments tends to evaluate function-based performance and client factors in the context of specific occupations. AOTA's centennial vision has leaded a rising demand for practitioners to provide evidence-based treatment to maximize effectiveness of client management ${ }^{15,16}$.

The assessment strategies used by OTs may be top-down or bottom-up approaches with respect to competency. The top-down approach is client oriented that focuses on its participation in day to day activities and also identify what is significant and related to the client while on the other hand, bottom-up approach examines small and discrete components occupational performance and skills, and the focus is on body functions and deficits ${ }^{17}$.

Studies shows that occupational therapist frequently use different cognitive assessment tools during their practice. In a recent study, 323 occupational therapists from different regions of the world were selected for the interview. These therapists had wide experience of working with clients of neurocognitive impairments. Results indicated that use of standardized cognitive assessment tools across the globe is inconsistent. A large proportion of therapist use non-cognitive specific assessment tools with focus on functional skills. Canadian Occupational Performance Measure was found to be the most common tool used by the therapist across the world followed by Mini Mental State Exam (MMSE) and Montreal-Cognitive Assessment (MoCA), while on the other hand, and non-standardized assessment include clinical observation (38.4\%) and Geriatric ADL Assessment (34.1\%). ${ }^{18}$ Similarly an another study also identified MMSE and MoCA have the strongest psychometric properties for evaluation of adults in acute care ${ }^{19}$ however, in another

This is an open-access article distributed under the terms of the Creative Commons Attribution License (CC BY) 4.0 
article it was found that MoCA is less sensitive in detecting mild cognitive impairment ${ }^{20}$.

In another study, it was found that informal assessments (clinical observation and interview) were most commonly used by occupational therapist in acute care setting. Standardized assessment tools were frequently used in complex situation $^{21}$.

Careful study of literature reveals that interviews and qualitative thematic method were widely used. It was indicated that occupational therapist working in acute hospital facility was interviewed regarding the use of specific assessments used in practice for the assessment of motor planning and skills. Although, it was observed that occupational therapist find it challenging to implement client-centered and occupation-based assessment tools into clinical practice.

A very few studies have been conducted in Pakistan to study selection of standardized and non-standardized assessment tools used by occupational therapist of Pakistan to evaluate cognitive abilities. Therefore, the aim of this study is to identify the assessment tools used by pediatric occupational therapist, and to determine the significance of standardize tool in occupational therapy practice during assessment and evaluation for effective intervention plan.

\section{METHODOLOGY}

Study Setting: The study was conducted at out-patient settings of Occupational Therapy Departments of primary and tertiary care hospitals, Pediatric rehabilitation clinics and special need schools.

Targeted Population: Pediatric Occupational Therapists

Study Design: Exploratory study.

Duration of Study: 6 months.
Sampling Technique: Non-Probability Convenience Sampling Technique. The sample size was calculated using online software open EPI, open source calculator version 3.01. Considering 100000 population with $50 \%$ of anticipated frequency, therefore a sample of 150 was calculated at $95 \%$ Confidence Interval.

\section{Inclusion criteria}

Occupational Therapists working with experience of $>1$ year at pediatric setting working with clients mild to moderate cognitive impairment.

\section{Exclusion criteria}

Occupational therapist working in acute care setting, developmental screening setup, and community based rehabilitation and physical disability only.

Data Collection Procedure: Data was collected from Occupational Therapists who were working in rehabilitation department using convenience sampling technique.

\section{Ethical consideration:}

Prior to the data collection, consent were taken to assure their confidentiality of participation. Further the details related to the study were also explained. Following their consent, self-administered questionnaire was given to the therapists related to the evaluation of tool they use for patients with cognitive impairments.

Data Collection Tool: Data was collected through a self-structured questionnaire which was validated by factor analysis through SPSS. It comprised of two parts:

(1) Demographic factors of the respondent.

(2) Assessment of clients with cognitive impairments consisting of 7 items.

All the questions are multiple choice based except for the 7th question, for the 7th question the respondents have options of 
choosing up to four alternatives. All the alternatives are not defined so respondents will answer the question with related definition in their mind. The estimated time for the completion of questionnaire is 4-5 minutes respectively.

Data Analysis Strategy: Data was entered and analyzed on SPSS (Statistical Package for Social Sciences). Participant's demographic details were represented through descriptive statistics whereas participant's responses on questions were represented through frequency and percentage.

\section{RESULTS}

A total of 150 participants enrolled in the study from sample included 18 males and 132 females. The participants have mean age of $27 \pm 3.61$ years with experience of $2.2 \pm 1.2$ years. $12 \%$ of the occupational therapists have deal with one client group while $73.5 \%$ deal with multiple groups as shown in Table-1.

It was reported that $96 \%$ of occupational therapist performed cognitive assessment while $4 \%$ did not (Figure-1). It is also noted that $9.6 \%$ of the therapist administer standardized assessments while $3.6 \%$ use clinical observation, $2.4 \%$ use conversation with the client and family, $1.2 \%$ semistructured interview and $2.4 \%$ performed structured interview to evaluate cognitive abilities (Figure-2). Furthermore, it was reported that $19.3 \%$ used cognitive tools for effective findings, $18.1 \%$ to start the intervention (Figure-3) and $4.8 \%$ therapist use standardized assessment tools to assess the impact of intervention. The present study also revealed that around $10 \%$ of the occupational therapist does not use standardized cognitive assessment tools due to incompetency, lack of resources and shortage of time. MMSE is found to be most common cognitive assessment tool while LOCTA is used least by the therapists in the present study.

\section{FIGURE-1: Cognitive Assessment by OTr}

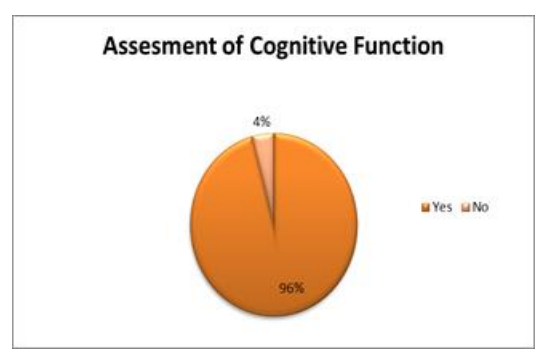

Figure-2: Methods used for cognitive assessment

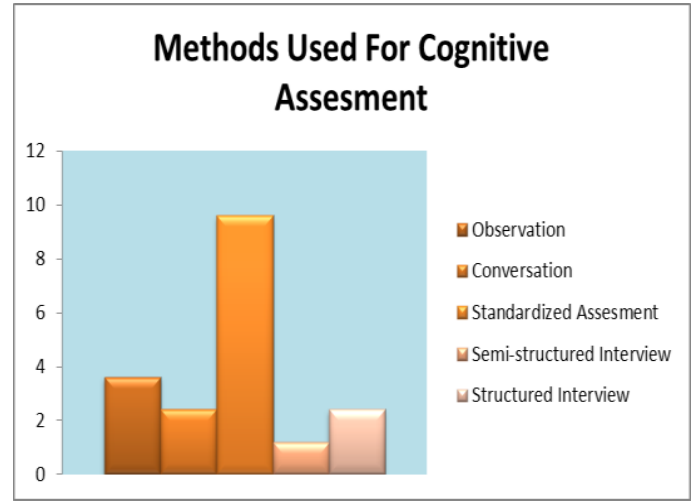

Figure-3 Reasons for using standardized tool

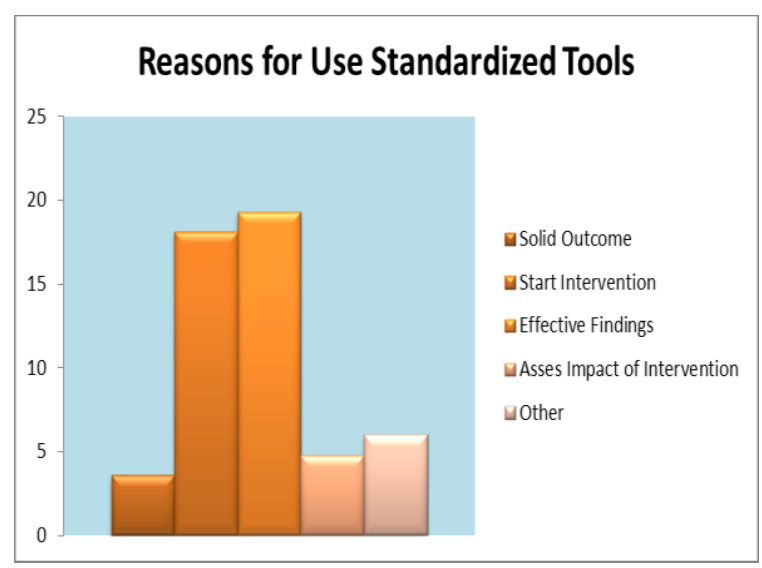

Figure-4 Reasons for using standardized tools 

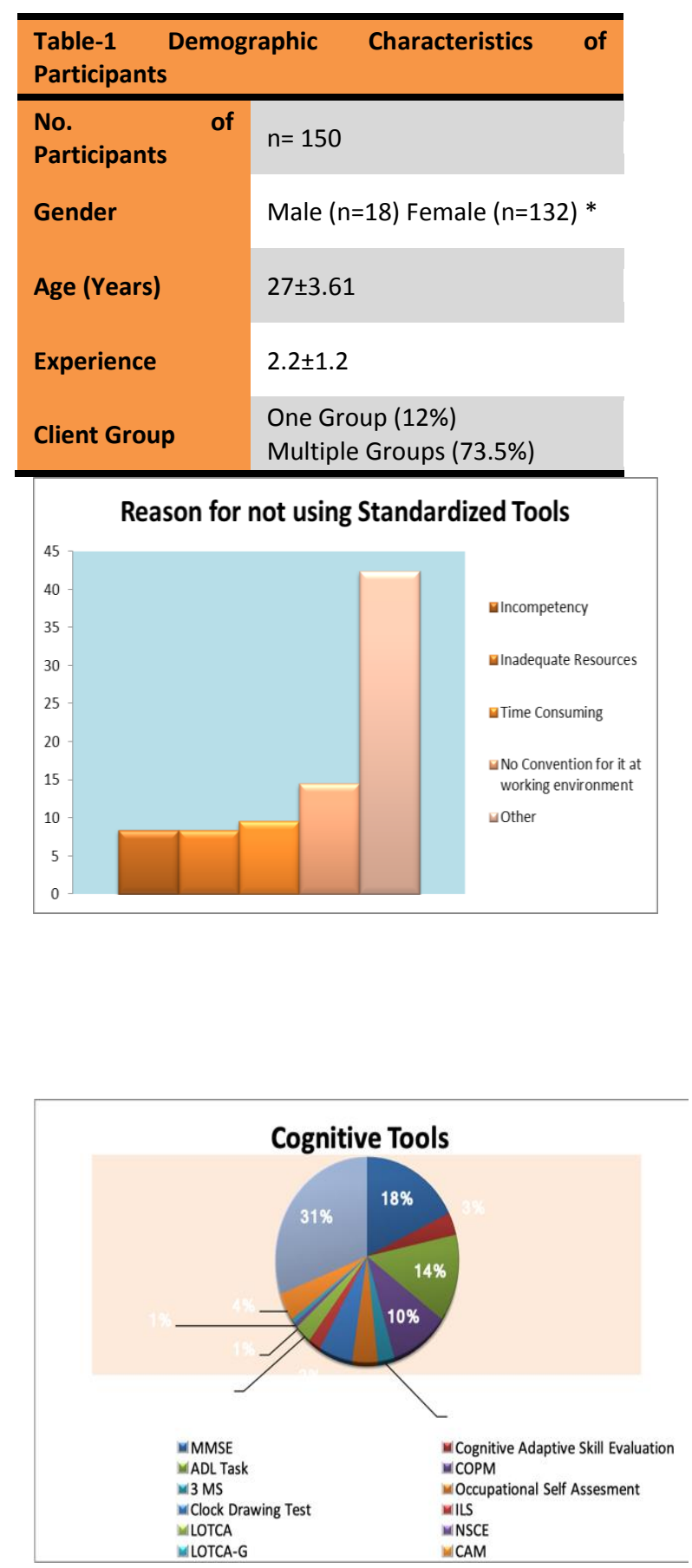

Figure-5 Cognitive tools used for OTs

\section{DISCUSSION}

The present study revealed the proportion of occupational therapist in Pakistan that use cognitive assessment tools during their clinical practice, identified the commonly used cognitive tools and reasons for standardized and non-standardized assessment during clinical practice. The main purpose of this study was to evaluate the significance of cognitive assessment in relation to pediatric occupational therapy practice and analyze the gaps in administration of standardized assessments.

The findings of this study revealed that $96 \%$ of occupational therapist performed cognitive assessment while $4 \%$ did not. $9.6 \%$ of occupational therapists were found to administer standardized assessments while others use informal methods of client assessment and evaluation that includes observation (3.6\%), conversation with the client and care taker (2.4\%), semi-structured interview (1.2\%) and structured interview (2.4\%. Furthermore, the present study highlights that the therapists used standardized cognitive assessment tools for variety of reasons, $19.3 \%$ of the occupational therapists under study used cognitive tools for effective findings and $18.1 \%$ to start the intervention while $14.5 \%$ find it no convention to use the tools in working environment whereas other found it not applicable to use.

Upon careful analysis of the results, it was found that observation is the most commonly use method by the therapists that accounted for $58 \%$. This finding was consistent with the study of (Victor et al $(2019)^{22}$ and Brown and Carrol $(2016)^{23}$ which highlighted that occupational therapists utilize informal observations more often compared to formal assessments in different type of clinical settings. Moreover, conversation accounted for $46 \%$ this finding is consistent with the study of Robertson and Btaga (2013) that shows that informal assessment method i.e. conversation and observation were more frequently used assessment method while standardized assessment method accounted for $46.25 \%{ }^{24}$, however another study showed that OTs in pediatric clinical setting reported that they use standardized assessments more frequently than non- 
standardized assessment method. (PiernikYoder and Beck, 2012) ${ }^{25}$.

It was also revealed by the results of present study that MMSE is the most commonly used standardized assessment tool in OT practices which is consistent with the study of $\mathrm{Yu}$ et al (2018) that suggested MMSE was reported to have high internal consistency for clients with cognitive impairments. ${ }^{26}$ It was also observed in our study that Canadian Occupational Performance Measure is also frequently used by the therapist in their clinical practice. Another study revealed that COMP has the high test re-test ability (Yang et al, 2017) ${ }^{27}$. It was further emphasized by Dungen (2019) to promote OT evidence-based practice. The increasing prevalence of cognitive deficits due to brain injuries and aging has brought cognitive assessment and screening to the cutting edge. Further studies are needed to analyze the usage of cognitive tools in the assessment of cognitive deficits.

One of the study recommended that LCOTA is one of the comprehendible and easily to administered tool that is reliable and a valid test for The LOTCA seems to be a reliable and valid test for prelim initial evaluation of cognitive skills required for basic functions. ${ }^{28}$ More over it only takes 30 minutes which is quite appropriate for a child to focus during session. One of the study also indicated that there is a lot space in LCOTA for cultural diversity ${ }^{29}$ that means it can be implemented in context to Pakistan culture too.

The findings of the present study have highlighted the crucial aspect of occupational therapy clinical practice in Karachi-Pakistan, and it has also laid foundation for future studies about diversity in occupational therapy practices and clinical reasoning. Such studies are important as they aid in improving the clinical practice and client care. This research study has laid better and reliable foundation for the treatment plan of the clients in order to improve Occupational Therapy services for their clients and have better health outcomes. Furthermore, this study has highlighted the domain for the future researches in the Pakistan that will increase scope of Occupational Therapy interventions in rehabilitation. It has also highlighted the gaps in administration of standardized assessments in Pakistan. Considered actions are needed to take on the identified results to meet the gap and improve client care.

All the occupational therapist enrolled in the study practice in Karachi. The inclusion of therapist from other cities of Pakistan can provide more insight of occupational therapy practices. The other factor that brings limitation to the present is absence of acute care occupational therapist. All the occupational therapist enrolled in the study practice in out-patient clinical setup. Inclusion of therapist working in acute care in the future study can reveal better results.

\section{CONCLUSION}

It can be concluded from the present study that majority of the occupational therapist perform cognitive assessment at different stages of OT process, and most of them relies on non-standardized and informal methods of evaluation. Incompetency, lack of resources and time were identified as limiting factors in administration of standardized assessments.

\section{REFERENCE}

[1] Nolen-Hoeksema S, Fredrickson B, Loftus GR, Lutz C. Introduction to psychology. Cengage Learning; 2014.

[2] Pizzi MA, Richards LG. Promoting health, well-being, and quality of life in occupational therapy: A commitment to a paradigm shift for the next 100 years. American Journal of Occupational Therapy. 2017 Jul 1;71(4):7104170010p1-5. 
[3] Creek J, Lougher L. Occupational therapy and mental health. Elsevier Health Sciences; 2011 Nov 29.

[4] Jäncke L. Sex/gender differences in cognition, neurophysiology, and neuroanatomy. F1000Research. 2018;7.

[5] Dhakal A, Bobrin BD. Cognitive Deficits. StatPearls [Internet]. 2020 Jun 28.

[6] Morley JE, Morris JC, Berg-Weger M, Borson S, Carpenter BD, Del Campo N, Dubois B, Fargo K, Fitten $\amalg$, Flaherty $\mathrm{JH}$, Ganguli $M$. Brain health: the importance of recognizing cognitive impairment: an IAGG consensus conference. Journal of the American Medical Directors Association. 2015 Sep 1;16(9):731-9.

[7] Kim BJ, Liu L, Cheung C, Ahn J. Effects of cognitive impairment and functional limitation on depressive symptoms among community-dwelling older Korean immigrants in the US. PloS one. 2018 Mar 26;13(3):e0193092.

[8] Kim KU, Kim SH, Oh HW. The effects of occupation-centered activity program on fall-related factors and quality of life in patients with dementia. Journal of physical therapy science. 2017;29(7):1188-91.

[9] Rosli R, Tan MP, Gray WK, Subramanian $P$, Chin AV. Cognitive assessment tools in Asia: a systematic review. International psychogeriatrics. 2016 Feb 1;28(2):189.

[10] De Roeck EE, De Deyn PP, Dierckx E, Engelborghs $S$. Brief cognitive screening instruments for early detection of Alzheimer's disease: a systematic review. Alzheimer's research \& therapy. 2019 Dec;11(1):14.

[11] Tavares-Júnior JW, De Souza AC, Alves GS, Bonfadini JD, Siqueira-Neto JI, Braga-Neto P. Cognitive assessment tools for screening older adults with low levels of education: a critical review. Frontiers in psychiatry. 2019 Dec 13;10:878.
[12] Sleutjes DK, Harmsen IJ, van Bergen FS, Oosterman JM, Dautzenberg PL, Kessels RP. Validity of the Mini-Mental State Examination-2 in Diagnosing Mild Cognitive Impairment and Dementia in Patients Visiting an Outpatient Clinic in the Netherlands. Alzheimer disease and associated disorders. 2020 Jul;34(3):278.

[13] Li KY, Lin LJ. Minimal clinically important difference of the loewenstein occupational therapy cognitive assessment-geriatric (LOTCA-G) in people with dementia. American Journal of Occupational Therapy. 2020 Nov 1;74(6):7406205020p1-7.

[14] Abreu BC, Toglia JP. Cognitive rehabilitation: a model for occupational therapy. American journal of occupational therapy. $1987 \mathrm{Jul}$ 1;41(7):439-48.

[15] Asaba E, Nakamura M, Asaba A, Kottorp A. Integrating Occupational Therapy Specific Assessments in Practice: Exploring Practitioner Experiences. Occupational therapy international. 2017;2017.

[16] Wood TJ, Wolgemuth K. Top-Down vs. Bottom-Up: A Case Series in Verbal Working Memory Treatments for Chronic Traumatic Brain Injury Deficits. Perspectives of the ASHA Special Interest Groups. 2019 Oct 31;4(5):1199-213.

[17] Brown C, Carroll J. Use of Assessments in Occupational Therapy Physical Disability Settings.

[18] Manee FS, Nadar MS, Alotaibi NM, Rassafiani M. Cognitive Assessments Used in Occupational Therapy Practice: A Global Perspective. Occupational Therapy International. 2020 Aug $12 ; 2020$.

[19] Arviso CC. Comparison of Adaptive Online Education with Adaptive Traditional Face-to-Face Education at Navajo Technical University: A 
Quantitative Study (Doctoral dissertation, Northcentral University).

[20] Harrison J, Lenk S, Logan B. Cognitive and Occupationally-based Assessments in Acute Care: For Individuals with Acquired Brain Injury.

[21] Conti J. Cognitive assessment: A challenge for occupational therapists in Brazil. Dementia \& neuropsychologia. 2017 Jun;11(2):121-8.

[22] Victor, C., Krutis, S., Chandler, E., Chang, B., Cherry, W., Doughty, P., Hubka, D., Murphy, K. and Zepf, Z., 2019. Perspectives of OTs Regarding Use of Standardized Assessments To Distinguish Levels of Evaluation in a Skilled-Nursing Facility. American Journal of Occupational Therapy, 73(4_Supplement_1), pp.7311505172p1-7311505172p1.

[23] Brown, C. and Carroll, J., 2016. Use of Assessments in Occupational Therapy Physical Disability Settings.

[24] Robertson, L. and Blaga, L., 2013. Occupational therapy assessments used in acute physical care settings. Scandinavian journal of occupational therapy, 20(2), pp.127-135.

[25] Pilegaard, M.S., Pilegaard, B.S., Birn, I., Kristensen, H.K. and Morgan, M.F., 2014. Assessment of occupational performance problems due to cognitive deficits in stroke rehabilitation: A survey. International Journal of Therapy and Rehabilitation, 21(6), pp.280-288

[26] Yu, S.T.S., Yu, M.L., Brown, T. and Andrews, H., 2018. Association between older adults' functional performance and their scores on the Mini Mental State Examination (MMSE) and Montreal Cognitive Assessment (MoCA). Irish Journal of Occupational Therapy.

[27] Yang, S.Y., Lin, C.Y., Lee, Y.C. and Chang, J.H., 2017. The Canadian occupational performance measure for patients with stroke: a systematic review. Journal of physical therapy science, 29(3), pp.548-555.

[28] Rojo-Mota G, Pedrero-Pérez EJ, Ruiz-Sánchez de León JM, LeónFrade I, Aldea-Poyo P, AlonsoRodríguez $\mathrm{M}$, Pedrero-Aguilar J, Morales-Alonso S. Loewenstein occupational therapy cognitive assessment to evaluate people with addictions. Occupational therapy international. 2017 Jan 10;2017.

[29] Almomani F, Avi-Itzhak T, Demeter N, Josman N, Al-Momani MO. Construct validity and internal consistency reliability of the Loewenstein occupational therapy cognitive assessment (LOTCA). BMC psychiatry. 2018 Dec;18(1):1-9. 